\title{
II Sección:
}

Educación y estrategias metodológicas

\section{Propuesta Teórico Metodológica para el abordaje de Estudios por Medio del Enfoque la Historia Oral}

\author{
Jorge Bartels Villanueva \\ Universidad de Costa Rica, Costa Rica \\ jorge.bartels@ucr.ac.cr \\ https://orcid.org/0000-0002-5530-2423
}

Recibido: 6 de agosto de 2019

Aceptado: 28 de setiembre de 2019

Resumen: Este artículo presenta la metodología construida y utilizada en el proyecto de investigación denominado "La re-construcción del paisaje de Puntarenas y Limón, como centros históricos del turismo costarricense, a partir de fuentes orales. 1930-2012"1. Con esta metodología se recopiló la información necesaria para realizar una interpretación de los espacios turísticos tradicionales de Costa Rica desde la perspectiva de sus habitantes.

Interesa en este caso reconstruir el paisaje de una forma vivencial, posibilitar la recreación de esos paisajes desde el punto de vista de sus actores, los cuales en muchos casos no tienen conciencia de las transformaciones, simplemente las han asumido y los más jóvenes no tendrán mayor referencia de esas estas.

Consideramos que se constituye en un pequeño aporte metodológico que puede ser replicado en otros espacios y con otras temáticas.

Palabras claves: Historia oral, metodología, Puntarenas, paisaje, entrevista, medios tecnológicos.

\section{Theoretical Methodological proposal for the approach of studies through the oral history approach}

\footnotetext{
${ }^{1}$ El proyecto "Centros Históricos del Turismo: Puntarenas y Limón", fue desarrollado por docentes de la Escuela de Sociología y las sedes del Pacífico y del Caribe de la Universidad de Costa Rica. Fue inscrito en la Vicerrectoría de Investigación de la Universidad de Costa Rica, gracias a que recibió el aporte del fondo de estímulo a la investigación INTERSEDES. El equipo de trabajo estuvo integrado por Susan Chen, Jorge Bartels, Pablo Carballo, Ivonne Lepe, Bladimiro Argueta, Cynthia Arrieta y Diana Guardia.
}

\section{(c) (i) (2)}

La Revista Estudios es editada por la Universidad de Costa Rica y se distribuye bajo una Licencia Creative Commons Atribución-NoComercial-CompartirIgual 3.0 Costa Rica. Para más información envíe un mensaje a 
Abstract: This article presents the constructed and used methodology on the investigation project called "The reconstruction of the landscape of Puntarenas and Limón, as historical centers of the Costa Rican tourism, from oral sources. 19302012." The information needed to fulfill an interpretation of the Costa Rican tradicional tourist spaces from its locals' perspectives was collected with this methodology.

In this case, it is of relevant interest to reconstruct the landscape in a experiential way. This to make possible the most completed recreation of such landscapes from its locals' point of view, who most of times are not aware of the different transformations and had only assumed them, so that the youngest people are not going to have any references to it.

We considered that it is established in a small and methodological input that could be retorted in different spaces with distinct thematics.

Keywords: Oral history, methodology, Puntarenas, landscape, interview, technological means.

\section{1.- El problema y su justificación}

Tanto en Puntarenas como Limón, la actividad portuaria, constituye el eje principal de la economía local. Igualmente es por estos puertos donde se realiza en gran parte el relacionamiento internacional de la economía costarricense. Por muchos años, el empleo generado por la actividad portuaria fue público. Esto ya no es así en Puntarenas, mientras que en Limón es objeto de una crisis socio-política probablemente solo comparable con la ida de la Compañía Bananera. De manera que el tema central para el futuro de estos lugares, es de qué manera pueden encontrarse nuevos espacios de empleo y por tanto de cierta realización social, si es que se puede hablar en estos términos. Es en tal marco, donde el tema del modelo de turismo posible y deseable es de mucha importancia. El tema de las posibles alternativas de empleo, incluyendo el modelo turístico deseable, es independiente de la resolución que tenga finalmente la crisis del empleo público. Obviamente, tal problemática no es solo importante desde un punto de vista académico, sino que es vital para significativos sectores sociales de estos lugares.

\section{(c) (i) (2) (2)}

La Revista Estudios es editada por la Universidad de Costa Rica y se distribuye bajo una Licencia Creative Commons Atribución-NoComercial-CompartirIgual 3.0 Costa Rica. Para más información envíe un mensaje a 
Es por ello que es importante recurrir a las fuentes orales, para conseguir la información que nos permita comprender como interpretan los actores sociales, hombres y mujeres, de distintas edades, clases sociales, posición económica, condición política o actividad económica, el paisaje que le ha correspondido vivenciar, y como interpretan su propia participación en la transformación del mismo.

Este enfoque metodológico se pretende construir mediante un ejemplo que nos permita comprender los paisajes evolutivos (u orgánicamente desarrollados), asociados con la actividad turística, resultantes de condicionantes sociales, económicas, administrativas, políticas y religiosas, que se han desarrollado conjuntamente y en respuesta a su medio ambiente natural. Esta última categoría de paisajes es propuesta por la UNESCO.

Para el estudio se parte del hecho de que los paisajes son "construidos (y destruidos) continuamente por la humanidad, siendo la economía el principal factor de (re)configuración de dichos paisajes. En términos de acción humana, la categoría "trabajo" sería la principal determinante social de la (re) creación de los paisajes" (Allen, 2014, p.16)

En el caso del turismo, el paisaje, en particular los paisajes locales, se recrean a través de manifestaciones como las siguientes:

- Revalorizando ciertos paisajes donde el trabajo fue constitutivo de los mismos.

- Desvalorizando otros paisajes en función de la "evolución" de los gustos turísticos y las tendencias del mercado.

- Creando nuevos paisajes donde el ocio y la recreación son sus fuerzas constituyentes. Igualmente son importantes, los propios paisajes del ocio, construidos por los propios pobladores locales. El turismo, tiende a re-utilizar y por ende a resignificar los espacios originarios del ocio.

\section{(c) (i) (2)}

La Revista Estudios es editada por la Universidad de Costa Rica y se distribuye bajo una Licencia Creative Commons Atribución-NoComercial-CompartirIgual 3.0 Costa Rica. Para más información envíe un mensaje a 
Revista Estudios, (39), 2019.

Diciembre 2019-Mayo 2020

ISSN 1659-3316

Bartels Villanueva Jorge

En los casos que aquí nos ocupan, que es el de los principales puertos de Costa Rica, Puntarenas y Limón, destacan como articuladores de los paisajes socioculturales:

- El transporte ferroviario y acuático; incluyendo sus puertos.

- Los mercados, específicamente los mercados centrales.

- Los paisajes étnicos.

- Los paisajes del ocio y la recreación de cada uno de los puertos.

"Por lo tanto lo que interesa es realizar una re-construcción histórica de los paisajes asociados a esos espacios para comprender su evolución y como han impactado al turismo en las zonas en estudio".(Allen, 2014, p.17)

El periodo de estudio comprende de 1930 hasta el año 2013, debido a que sería difícil encontrar informantes con edades superiores s los 80 años en condiciones de salud que les permitan proporcionar información con la facilidad que se requiere. Además este periodo permite hacer una reconstrucción del paisaje que incorpora buena parte de los elementos que visibilizan los cambios más importantes ocurridos en esta zona.

La zona que se analizará es lo que se conoce como el cantón de Puntarenas, que incluye tanto lo que se ha denominado en centro o "Puerto", como los lugares aledaños, esto porque en muchas ocasiones los pobladores del puerto venían procedentes de esos lugares vecinos, atraídos por las fuentes de trabajo, por razones de comercio o simplemente porque el puerto se constituía en el centro de atracción de la zona. Esto permite a la vez realizar un contraste entre el paisaje del puerto y de sus alrededores, el cual en ocasiones podría resultar muy distinto.

El objetivo general del proyecto es realizar una re-construcción del paisaje de Puntarenas y Limón que permita reposicionar el turismo como actividad productiva que genere desarrollo y que pueda corregir y evitar deficiencias del pasado y que la UCR les acompañe y les colabore en dicho proceso.

El paisaje como activo cultural requiere de un adecuado manejo del territorio, considerando que en él hay evidencias particulares e irremplazables que deben

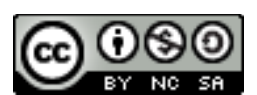

La Revista Estudios es editada por la Universidad de Costa Rica y se distribuye bajo una Licencia Creative Commons Atribución-NoComercial-CompartirIgual 3.0 Costa Rica. Para más información envíe un mensaje a revistaestudios.eeg@ucr.ac.cr. 
ser protegidas, entendiendo que el espacio que las alberga es dinámico y constantemente cambiante, lo que constituye un potencial para las poblaciones aledañas, no solo en términos económicos (turísticos por ejemplo) sino sociales, por lo que la comprensión de su evolución y la participación de sus actores resulta vital.

Una investigación de este tipo tiene repercusiones a nivel de las ciencias sociales, ya que permitirá ver el producto de un equipo interdisciplinario trabajando un tema poco estudiando en el país y el resultado de las narraciones en las que la frontera entre lo que tiene lugar fuera del narrador y lo que sucede en su interior, entre lo que conocemos del individuo y lo que se refiere al grupo, puede ser más aleccionador inclusive que lo que encontramos en los géneros escritos establecidos, y nos puede iluminar sobre si la visión personal de los entrevistados coincidir con la visión oficial que compartimos.

\section{La oralidad como enfoque teórico metodológico para el desarrollo del proyecto}

La historia, al referirse a hechos del pasado, requiere que las fuentes de información con que trabaja sean lo más objetivas posibles, lo cual al devolvernos en el tiempo, la obtención de dichas fuentes, su claridad y su oportunidad se tornan cada vez de menor calidad.

La historia oral es una especialidad dentro de la ciencia histórica que se basa en los testimonios orales como su principal fuente para la reconstrucción del pasado y hasta del presente. El registro de los hechos se basa en la memoria y la transmisión oral.

Algunos autores clasifican la historia oral como una "técnica" que se usa para registrar y recuperar el conocimiento directo de un hecho histórico que posee la fuente, ya sea por medio de entrevistas, testimonios, y del cual fueron protagonistas directos. Por eso, se concentra en los procesos contemporáneos.

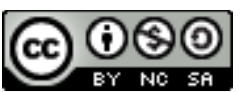

La Revista Estudios es editada por la Universidad de Costa Rica y se distribuye bajo una Licencia Creative Commons Atribución-NoComercial-CompartirIgual 3.0 Costa Rica. Para más información envíe un mensaje a revistaestudios.eeg@ucr.ac.cr. 
En este sentido, cuando se hace historia oral lo que se recogen son experiencias del pasado reciente, contadas desde la perspectiva del presente.

Cuando se hace referencia a fuentes escritas, nos encontramos que una gran mayoría de esta la documentación es generada por el Estado a través de sus organismos centrales y regionales, así como por entidades asociadas a él, por instituciones privadas y por los particulares, ha crecido exponencialmente respecto de los siglos anteriores. La propia creación de una institucionalidad que administra esa información, así como la aparición de numerosos periódicos locales y otras tantas instituciones productoras de documentos permiten acercarse a la historia de cada sociedad gracias a las colecciones de los mismos. Otra fuente de información histórica es dada por las fuentes de origen judicial, sobre todo aquellas de carácter penal recientemente comenzadas a utilizar por los historiadores.

Estas fuentes si bien son valiosas tienen una característica común: reproducen el discurso oficial, generalmente dominado por las elites nacionales y regionales, a los funcionarios o a los hombres ilustrados que se ocupaban de esos diarios de los entes estatales que se encargaban de generar y recopilar la información.

Las limitaciones que presentan las fuentes como los libros y documentos históricos, al no poder contar todo sobre nuestro pasado y limitarse a ciertos elementos que desea resaltar quien los produce, deja de lado, en la mayoría de las ocasiones, hechos y personas comunes, sus historias diarias, la historia de los excluidos o la historia de los vencidos (comunidades étnicas minoritarias, mujeres, marginados sociales, delincuentes, homosexuales, etc.).

Es así como, los pobres y las clases subalternas difícilmente aparece expresada directamente por ellos o, si eso sucede, lo hace, al menos, mediatizada por las la visión de dichos funcionarios o de los periodistas de los diarios de la época.

Es por esto que se ha considerado la historia oral como el recurso más valioso para recuperar lo que la "historia oficial" ha dejado de lado y permite recuperar

La Revista Estudios es editada por la Universidad de Costa Rica y se distribuye bajo una Licencia Creative Commons Atribución-NoComercial-CompartirIgual 3.0 Costa Rica. Para más información envíe un mensaje a revistaestudios.eeg@ucr.ac.cr. 
experiencias olvidadas o desconocidas, porque la particular forma en que la gente da sentido a sus vidas es una evidencia históricamente valiosa. Se trata de dar voz a los que no la tienen.

La historia oral permite entender por su propia cuenta otras realidades históricas y en los últimos tiempos se ha convertido en una herramienta útil para la explicación de una serie de fenómenos en campos como la historia social, cultural, regional o local, la historia ambiental y hasta en historia económica. Por medio de la historia oral nos encontramos las historias de vida familiares, las migraciones, costumbres, historia oral de los pueblos o la propia autobiografía. Es por eso que esta herramienta permite que la historia recobre su utilidad como ciencia, retomando el acervo común de la sociedad y no solo la historia oficial. Además con el paso del tiempo ha propiciado la renovación de las técnicas y métodos en el trabajo de investigación histórica, lo mismo que los recursos didácticos.

La historia oral, por medio de la aplicación de sus metodologías, permite el acercamiento interdisciplinario a las fuentes, de forma que un mismo objeto de estudio puede ser analizado por sociólogos, antropólogos, literatos, etc., al mismo tiempo, lo que permitirá una aprehensión del conocimiento de una manera integral. De esta manera, al descubrir fuentes vivas e involucrarse con ellas permite trascender el estudio de las fuentes y objetos inanimados.

Entre las disciplinas que han hecho uso de la historia oral y que han aportado en su desarrollo epistemológico, aún antes de la aparición de la grabadora están la psicología y el psicoanálisis en los procesos de comunicación y el papel que cumple el mismo investigador en el proceso de creación de conocimientos, en relación al inconsciente. Los literatos, lingüistas y folcloristas han contribuido con técnicas de recolección de información oral, procedimiento metodológico y técnicas de formalización de textos recuperados. Estas contribuciones permitieron el rescate de la tradición oral popular.

\section{(c) (i) (-)}

La Revista Estudios es editada por la Universidad de Costa Rica y se distribuye bajo una Licencia Creative Commons Atribución-NoComercial-CompartirIgual 3.0 Costa Rica. Para más información envíe un mensaje a 
De modo que al hablar de la historia oral como un método de investigación, nos estamos refiriendo al procedimiento establecido de construcción de nuevas fuentes para la investigación sociohistórica. Con base en los testimonios orales recogidos sistemáticamente para investigaciones específicas, bajo métodos, problemas y puntos de partida teóricos explícitos (Thompson. 1988. p. 268).

La historia oral ha cobrado importancia a nivel mundial y aún está en proceso de formación, por lo que esta actividad requiere que se constituya un corpus abundante y significativo de trabajo historiográfico, con base en la construcción de y empleo de fuentes orales; materia prima del historiador oral: que se han clasificado como elementos subjetivos: memoria, recuerdo, testimonio etc. $Y$ esto permite realizar una reconsideración de la historia oral, ya que en los orígenes del saber histórico se emplearon testimonios de viva voz.

Como bien señalan Barela, Miguez y García, la historia oral ofrece posibilidades para hacer la historia de construcciones (religiosas, educativas, sanitarias, una industria o actividad local, etc.), de una institución (escuela, club, hospital), de la cuadra, del barrio o del pueblo. También encontramos propuestas de investigación en torno de un acontecimiento local importante, un hecho de fuerte impacto social, el cierre del ferrocarril o de una industria, o bien, la repercusión local de un acontecimiento nacional o internacional. (Barela, Miguez y García, 2004, p. 31.)

Desde el proyecto de Centros Históricos del Turismo se trabaja en las ciudades de Puntarenas y Limón, partiendo de las distintas temáticas ya mencionadas que involucran cambios en el paisaje como serían el asfaltado de las calles, la aparición de servicios públicos, la educación, la condición de sectores de actividad económica, la historia de algunas instituciones representativas, los carnavales, los sitios de recreación y esparcimiento. Es decir, las distintas características y maneras de interpretar los cambios en el paisaje regional de estos dos puertos.

\section{(C) $(00$}

La Revista Estudios es editada por la Universidad de Costa Rica y se distribuye bajo una Licencia Creative Commons Atribución-NoComercial-CompartirIgual 3.0 Costa Rica. Para más información envíe un mensaje a 
Revista Estudios, (39), 2019.

Diciembre 2019-Mayo 2020

ISSN 1659-3316

Bartels Villanueva Jorge

En nuestro caso, el intento consiste en realizar una propuesta de enfoque metodológico para abordar el estudio de hechos históricos, de diversa naturaleza, asociados al paisaje de Puntarenas y sus alrededores, desde el instrumental que utiliza la historia oral.

\section{Metodología a desarrollar}

Para realizar el estudio se utilizaran fuentes orales que serán seleccionadas por medio de un sondeo previo que permita identificar a informantes claves, que incluyan hombres y mujeres, con edades comprendidas entre los 15 y más años, hasta donde las facultades físicas y mentales de los posibles entrevistados lo permitan.

Es de nuestro interés contar con la visión de personas mayores, pero también de personas adultas y de jóvenes que puedan dar su interpretación de cómo reconocen el paisaje en que han vivido y las transformaciones que han percibido. Este elemento a la vez permite contrastar la visión de personas de diferentes edades y poder determinar cómo se asimilan esos cambios, sus impactos y el reconocimiento de su propia participación en la modificación de esos espacios.

En nuestro caso se pretende construir la historia oral en relación con los sectores subalternos, pero se no necesariamente renunciamos a entrevistar a personas de otros tipos de sectores, políticos por ejemplo, que permitan la comprensión de las trasformaciones del paisaje, contrastándolo con las fuentes subalternas. Esto es importante ya que nos se cuenta con información de este tipo.

Una condición necesaria de los informantes es que procedan de Puntarenas o de sus alrededores, es decir de lugares pertenecientes al cantón central de Puntarenas, no necesariamente deben ser oriundos de la zona, pero sí que hayan vivido la mayor parte de su vida en la zona. Esto para eliminar a informantes con un conocimiento relativamente escaso de la evolución del paisaje.

\section{(๑) $\odot \odot$}

La Revista Estudios es editada por la Universidad de Costa Rica y se distribuye bajo una Licencia Creative Commons Atribución-NoComercial-CompartirIgual 3.0 Costa Rica. Para más información envíe un mensaje a revistaestudios.eeg@ucr.ac.cr. 
Por otra parte se requiere que los informantes tengan relación con alguna de las actividades que se consideran más relevantes en el desarrollo de la zona como son el transporte ferroviario y acuático; incluyendo sus puertos, los mercados, específicamente los mercados centrales, los paisajes del ocio y la recreación del puerto. Es por ello que tiene importancia como fuentes de información, los pescadores, los ex trabajadores ferroviarios, los trabajadores portuarios, los políticos, educadores, comerciantes y otras personas relacionadas con las actividades de ocio, sean tanto del turismo como relacionados con este tipo de actividades y por último de jóvenes, estudiantes y otros que no se hayan vinculado a los estudios formales. Esto no quiere decir que un sujeto no relacionado con estas actividades no pueda ser considerado, sobre todo si es referenciado por alguna otra fuente que considere importante la información que nos pueda proporcionar.

La información que se busca es aquella que permita comprender la forma como se ha trasformado el paisaje de Puntarenas $^{2}$ a lo largo del periodo de estudio, visto no desde la perspectiva de Puntarenas como puerto comercial y como destino turístico para los habitantes del resto del país, sino como lugar de existencia, de coexistencia, de trabajo y de desarrollo de políticas públicas que los afectan directamente y que inciden en sus vidas, y como ellos mismos han sido participes de esos cambios y de los impactos que les han afectado. En este sentido la información recopilada a partir de personas jóvenes adquiere gran importancia al permitir contar con información sobre la visión de los impactos más recientes, y como esa población asume su condición actual y las perspectivas a futuro.

A partir de la información proporcionada por los entrevistados se pretende hacer un cruce, que permita hacer la valoración de las categorías a estudiar (producción, transporte, comercio, ocio), desde la visión de informantes con distintos roles en la

\footnotetext{
${ }^{2}$ Me refiero a Puntarenas por ser parte del equipo que trabajo en este zona, sin embargo, la propuesta debería desarrollarse de la misma forma en ambas comunidades.

\section{(c) (i) (-)}

La Revista Estudios es editada por la Universidad de Costa Rica y se distribuye bajo una Licencia Creative Commons Atribución-NoComercial-CompartirIgual 3.0 Costa Rica. Para más información envíe un mensaje a revistaestudios.eeg@ucr.ac.cr.
} 
sociedad porteña, es decir, distintas profesiones, trabajos, edades y procedencia social.

La cantidad de informantes, para iniciar, se ha estimado en 12 personas, subdivididas en tres grupos de edad (jóvenes hasta 30 años, adultos hasta ó0 años y adultos mayores de 61 años y más) relacionados con de diferentes áreas de trabajo (inicialmente se trabajará con pescadores, políticos y personas relacionadas con el ocio) a razón de cuatro personas por cada uno de los grupos mencionados.

En relación con la actividad pesquera y la transformación del paisaje de la pesca lo que interesa es conocer cómo ha evolucionado una actividad que en esta zona ha tenidos y aún mantiene una gran importancia, constituyéndose en uno de los proveedores, por no decir el más importante, en el abastecimiento de los productos del mar para el país, que ha sido una importante fuente de empleo y que ha venido a menos por una serie de causas que han mermado la fuente del recurso. Es importante conocer de estos informantes como ven ellos este proceso y determinar como reconocen su propia participación en el mismo.

Por otra parte, las entrevistas a personas relacionadas con la política es importante para comprender como las políticas públicas, desarrolladas a lo interno, como las que se han propiciado desde fuera, han incidido en el paisaje de Puntarenas, en este caso relacionados con las actividades productivas, el desarrollo de infraestructura y las condiciones de crecimiento-desarrollo o subdesarrollo en que se ha sumido la zona. Aquí de nuevo es importante conocer cómo se asume la participación en las trasformaciones del paisaje tanto a lo externo, como de los mismos entrevistados. Esta información es fundamental para comprender aspectos culturales asociados a devenir de esta zona y comprender su estado actual.

\section{(c) (i) (-)}

La Revista Estudios es editada por la Universidad de Costa Rica y se distribuye bajo una Licencia Creative Commons Atribución-NoComercial-CompartirIgual 3.0 Costa Rica. Para más información envíe un mensaje a 
Por último, la información relacionada con el ocio estuvo asociada a Puntarenas como uno de los destinos preferidos para el turismo nacional durante muchos años, y como esta actividad económica se ha transformado, ha ido perdiendo intensidad y se ha visto sustituida por nuevos polos de atracción. Es importante ya que conocer la visión de los entrevistados sobre su relación con estas actividades y su impacto en el desarrollo del turismo y en las propias vidas de los pobladores permitirían conoce si estas actividades han tenido un impacto positivo o negativos sobre sus vidas y sobre el turismo de la zona.

Las principales limitaciones que se pueden encontrar con estas fuentes están asociadas a la disposición de participar de muchos de los posibles informantes, asociadas sobre todo al temor de ser entrevistados.

El desarrollo del proyecto será desarrollado por un grupo de investigadores integrado por docentes de las sede del Pacífico y del Caribe, así como de la Escuela de Sociología de la Universidad de Costa Rica, y está integrado por un total de 6 personas, es un equipo interdisciplinario. El tiempo para su desarrollo se estima en dos años calendario, que comprende las siguientes fases:

1. Exploración de campo para sondear los posibles informantes y levantar un directorio preliminar, tratando de que en este directorio participen personas involucradas con las diferentes actividades que se quiere investigar.

2. Confección de un directorio definitivo con la información detallada de los informantes, que incluye su localización, posibilidad de movilización, y calendario de realización de las entrevistas. También deben contemplarse informantes de reemplazo para el caso de que uno de los titulares no pueda ser entrevistado. El directorio debe ser acompañado de la ficha técnica, de forma que la información de cada informante ya se tenga contemplada en dicha ficha y solo sea completada en día de la entrevista.

3. Realización de las entrevistas en forma directa y a profundidad, que involucra la realización de las entrevistas en los lugares y fechas 
previamente definidos, el tratamiento de las entrevistas y un proceso de reflexión, por parte del equipo del proyecto, que permita medir el avance del proceso de recopilación y realizar la retroalimentación del mismo, de forma que se pueda corregir cualquier imprevisto que se presente.

4. La última fase comprende el proceso de sistematización y de divulgación de los resultados del proyecto, que debe involucrar tanto a los participantes como a las comunidades, de forma que se puedan cumplir los objetivos planteados y que la investigación tenga una aplicación práctica.

\section{La guía de entrevista a realizar}

Se ha planeado realizar entrevistas individuales, semi-estructurada con una serie de preguntas cuyo contenido y orden han sido preestablecidos, pero que permiten que las preguntas, modo y orden de enunciarlas, pueden ser variados por el entrevistador, según su criterio y cómo se vaya desenvolviendo la entrevista, para garantizar un orden en la recopilación de la información, a la vez que nos permitirá dar mayor seguridad al entrevistador y, además facilitará el análisis posterior de las entrevistas.

Las preguntas han sido elaboradas con base en una preparación previa, tomando como referencia los objetivos del proyecto, tratando que los entrevistados no se desvíen mucho del tema y buscando encausarlo de nuevo en caso de que se desvíen del mismo, pero sin perder sensibilidad hacia lo que el testigo quiere comunicar.

La guía de preguntas se elabora tratando de seguir un hilo conductor que permita obtener la información relevante de parte del entrevistado y que variará de acuerdo con la temática analizada.

\section{(c) (i) (-)}

La Revista Estudios es editada por la Universidad de Costa Rica y se distribuye bajo una Licencia Creative Commons Atribución-NoComercial-CompartirIgual 3.0 Costa Rica. Para más información envíe un mensaje a 
Existen varios elementos que deben ser tomados en cuenta, por el entrevistador, a la hora de la aplicación de las entrevistas para enfrentar posibles situaciones que se puedan presentar:

Los momentos de monotonía o silencio durante la entrevista: deben ser abordados con preguntas importantes que vuelvan al entrevistado sobre el tema.

El espacio donde se realice la entrevista: de forma que no se convierta en un lugar hostil para el entrevistado pero que a la vez cause el mínimo de interferencia durante las entrevistas, esto debe ser analizado antes de concertar las citas para la entrevista.

Estar preparado para apreciar las cuestiones potencialmente tensas o delicadas, evitando el tema si éste no es fundamental para la investigación.

El tiempo de duración de la entrevista: para lo cual se recomienda que no supere las dos horas, sin embargo es importantes no realizar un corte abrupto de la entrevista ya que en ese momento final se pueden presentar una información interesante que sea útil recabar.

Si la entrevista no se pueda terminar en el periodo establecido, se debe buscar la forma de concertar en ese momento un segundo encuentro; si esto no es así, es beneficioso dejar planteada esa posibilidad para el caso en que surja la necesidad luego de haber hecho un primer análisis del material recabado.

Por último es importante que como entrevistadores mantengamos una actitud de respeto, neutral con el informante, es decir, no entrar en discusión sobre sus puntos de opiniones, o intentar imponer nuestro propio juicio sobre un tema expuesto.

\section{Los medios tecnológicos a utilizar}

Para la recopilación de las entrevistas se utilizará prioritariamente el medio electrónico compuesto por grabadoras digitales que permiten la grabación de la entrevista y su posterior descarga o traslado a un computador para que pueda ser almacenada, escuchada o transcrita a un archivo de texto.

La Revista Estudios es editada por la Universidad de Costa Rica y se distribuye bajo una Licencia Creative Commons Atribución-NoComercial-CompartirIgual 3.0 Costa Rica. Para más información envíe un mensaje a revistaestudios.eeg@ucr.ac.cr. 
Revista Estudios, (39), 2019.

Diciembre 2019-Mayo 2020

ISSN 1659-3316

Bartels Villanueva Jorge

El uso de este tipo de medios requiere asegurase que estén en plena capacidad de funcionamiento y que las fuentes de energía para su funcionamiento nos permitan abarcar el tiempo planeado de la entrevista, de forma que no nos enfrentemos al entrevistado con una grabadora que no pueda ser usada y perdamos la oportunidad o le causemos una mala impresión al mismo.

Adicionalmente a la grabadora digital se puede elaborar una ficha técnica en papel, donde se anote el nombre del entrevistado, el lugar de la entrevista, fecha de la misma, y anotaciones que permitan recuperar situaciones claves que se producen durante la entrevista pero que no necesariamente quedan registradas en ella, de tal forma que puedan ser incorporadas o retomadas a la hora de analizar las entrevistas,

Alessandro Portelli, (citado en Barella et al, 2004.) señala que "la historia oral, a pesar de la omnisciencia de los medios electrónicos, continúa respetando la libertad de no querer contar, debido al gran respeto que ha desarrollado por los silencios. Es una vía, en la era de la globalización, para preservar la individualidad que no es lo mismo que el individualismo" (p. 14) de ahí que los apuntes que se puedan hacer durante la entrevista pueden constituir un buen elemento para rescatar la individualidad y los silencios de los entrevistados.

Tanto de la grabación digital como de la ficha técnica es necesario hacer la trascripción completa del material y de las notas de entrevista, aunque requiera de mucho tiempo y trabajo, pero que asegure contar con la información a la hora de realizar la sistematización de esta.

\section{(C) $(000$}

La Revista Estudios es editada por la Universidad de Costa Rica y se distribuye bajo una Licencia Creative Commons Atribución-NoComercial-CompartirIgual 3.0 Costa Rica. Para más información envíe un mensaje a revistaestudios.eeg@ucr.ac.cr. 


\section{El consentimiento informado}

Otro aspecto muy importante del estudio a realizar esta dado por el aspecto ético, que también es un elemento legal, porque se basa en los protocolos que regulan esta actividad, sobre todo en las Universidades y sirven para proteger tanto al entrevistado como al entrevistador, de la mala utilización de la información o de demandas legales por el uso de esa información.

Este tipo de instrumentos se comenzaron a utilizar en Estados Unidos por las demandas que se hacían a esas universidades, demandas legales que en ocasiones les representaron altos costos económicos. El consentimiento informado, y ahora institucionalmente acuerpado, previene de problemas que efectivamente lo lleven a los tribunales, por lo que pueda resultar de la información de las entrevistas.

\section{El procesamiento de la información}

a) El análisis: la formación del archivo oral.

Como bien explica Jorge Aceves, el proceso de análisis de cualquier material de investigación, recordemos, está estrechamente vinculado con los puntos de partida conceptuales, con los objetivos planteados, y con el método utilizado y con la serie de técnicas de instrumentos aplicados. El análisis no es, por lo tanto, una operación aislada de todo el proceso constitutivo de la evidencia empírica, sino que es más bien, el broche que amarra y une las diversas fases del proceso de la investigación". (Aceves, 1988, p.237)

$Y$ es por ello que en nuestro caso se propone realizar una tarea de reconocimiento de los textos orales recopilados por parte de todos los miembros del equipo, de forma que la información obtenida pueda ser analizada por cada uno de ellos sin importar la temática que esté abordando (pesca, política, ocio), y para ello se propone la elaboración de índices de contenidos por medio de la

\section{(@) $\odot \otimes \odot$}

La Revista Estudios es editada por la Universidad de Costa Rica y se distribuye bajo una Licencia Creative Commons Atribución-NoComercial-CompartirIgual 3.0 Costa Rica. Para más información envíe un mensaje a revistaestudios.eeg@ucr.ac.cr. 
clasificación y la codificación de los contenidos del todas las notas técnicas y grabaciones.

b) Ordenación, rotulación y clasificación del material recopilado.

Al igual que cuando se trabaja con fuentes escritas, el documento, producto de este tipo de metodología, es el la grabación que surge del encuentro, dentro del marco de una entrevista, entre el historiador y el informante. De esta forma cada archivo digital deberá ser almacenado en un medio magnético, como un disco compacto u un disco de video y deberá ser etiquetado con una reseña de la investigación de la cual surge, donde mínimamente figure el tema, las hipótesis, ciertas características de los entrevistados, como por ejemplo: edad, ocupación, lugar de residencia, o cualquier otro dato que contribuya a dar mayor y mejor sentido a lo que el consultante va a escuchar.

El coordinador del proyecto será el responsable del almacenamiento y custodia de los discos que contienen las entrevistas y deberá almacenarlos en condiciones ambientales y de seguridad, adecuadas, de forma que se pueda establecer un archivo y que el mismo esté abierto a la consulta pública, siempre y cuando los entrevistados no tengan reparo en ello y haciendo reserva de sus nombres o partes del documento si así lo exigieran.

Además de contar con el archivo, catalogado con el contenido de cada disco y las temáticas abarcadas en los documentos, se deberá tener una copia de respaldo de cada disco, y de las transcripciones textuales para ofrecer en su lugar o como complemento del audio. Esto por cuanto consideramos que es importante que otros investigadores puedan tener acceso a las fuentes de igual manera que lo hacen con la bibliografía o documentos existentes en cualquier archivo tradicional.

c) Elaboración de índices de contenido

Los índices de contenido para esta investigación están dados por las actividades que se pretende resaltar como modeladoras del paisaje de la zona, es decir de la 
Revista Estudios, (39), 2019.

Diciembre 2019-Mayo 2020

ISSN 1659-3316

Bartels Villanueva Jorge

pesca, la política, el ocio, el comercio, la etnia, etcétera, los cuales sirven para orientar la lectura de la experiencia particular de cada entrevistado y nos van a permitir ubicarlos en el contexto más amplio del cual forman parte, sí como de los aportes a los diferentes contenidos que se quiere analizar.

Además de los índices de contenido, se propone elaborar glosarios y cronologías específicas para cada informante dónde se resalte el tema principal sobe el cual aporto la información, un listado de temas secundarios mencionados y un esquemas biográficos donde se representen las experiencias aportadas por los entrevistados.

d) Clasificación y análisis del material

Con base en los índices de contenido, de los glosarios y de las cronologías es posible construir "una especie de matriz de experiencia individual, cronológica, que marque las líneas de flujo de la experiencia personal; o sea, los momentos de cambio, de crisis, de transición; matriz que puede organizarse por ámbitos de la experiencia: laboral, familiar, comunitaria, educativa, migración, política, religiosa, etcétera" (Aceves, 1988, p.238) Este se constituye en un trabajo preliminar y exploratorio que permite el acceso de forma más eficaz al contenido de la información de las entrevistas si necesidad de tener que recurrir a su revisión total y a la vez permite contar con "un catálogo muy preciso de todas las unidades narrativas, relatos personales, cuadros narrativos, trayectorias personales, géneros narrativos, etcétera, que se encuentran dispersos en todas las entrevistas". (Aceves, 1988, p. 239)

Esta matriz también es el requisito para cualquier estandarización o elaboración de algún tipo de muestra cualitativa representativa, ya que permite reconocer las reiteraciones, las oposiciones, las contradicciones, las diferentes versiones, los casos negativos, etcétera; además de favorecer y posibilitar, en un momento dado del proceso de recolección de los testimonios orales, la decisión de reconocer que

\section{(c) (i) (2)}

La Revista Estudios es editada por la Universidad de Costa Rica y se distribuye bajo una Licencia Creative Commons Atribución-NoComercial-CompartirIgual 3.0 Costa Rica. Para más información envíe un mensaje a 
Revista Estudios, (39), 2019.

ISSN 1659-3316

Diciembre 2019-Mayo 2020

Bartels Villanueva Jorge

se ha llegado al punto de saturación y que ya puede concluirse la recolección de la evidencia oral.

e) El proceso de trascripción de las cintas;

El paso de la información oral al texto es una operación necesaria e importante ya que de ello depende la calidad de nuestra investigación. La trascripción se puede realizar por parte del mismo entrevistador o por media otra persona que transcribe el material captado por la grabadora.

Lo que no es recomendable es atenerse a la memoria para luego hacer la transcripción, debemos recordar que el olvido aparece rápidamente, por lo que algunos autores recomiendan la toma de notas que pueden contribuir a la trascripción. Viviane Labrie, citada por Deslauriers, sugieren algunos principios para la trascripción de documentos orales:

“- El documento trascrito debe ser fiel a lo que dijeron los interlocutores y debe ser realizado con el respeto de las personas a las que concierne.

- En la trascripción, el documento debe presentar una experiencia oral directa.

- El documento trascrito debe reproducir el testimonio oral lo más fielmente posible.

- El transcriptor debe sujetarse al sentido transmitido y quitar toda ambigüedad creada por el pasaje a lo escrito.

- Una primera trascripción debe ser generosa y proporcionar amplias informaciones." (Deslauriers, 1991, p. 104).

Además, a la hora de realizar las transcripciones se trata de escribir correctamente distinguiendo el lenguaje utilizado, de manera simple y respetar la lógica del informante. Se debe respetar el sentido del entrevistado, realizando una buena

\section{(c) (i) (2)}

La Revista Estudios es editada por la Universidad de Costa Rica y se distribuye bajo una Licencia Creative Commons Atribución-NoComercial-CompartirIgual 3.0 Costa Rica. Para más información envíe un mensaje a revistaestudios.eeg@ucr.ac.cr. 
Revista Estudios, (39), 2019.

Diciembre 2019-Mayo 2020

ISSN 1659-3316

Bartels Villanueva Jorge

puntuación y concordancia verbal de forma que se pueda precisar el pensamiento pero sin transformarlo. En resumen, nuestra estrategia deberá orientarse a obtener el máximo de información útil.

f) Alternativas y opciones de análisis

Para el proceso de análisis de la información, se propone realizar tres etapas complementarias

1. Partir de la información, las evidencias y los testimonios provenientes de otras fuentes consultadas desde el arranque de 1a investigación. Para realizar este proceso se debe recurrir a la triangulación de fuentes, que consiste en conocer y considerar toda la información pertinente y relevante a nuestra investigación, procedente de los archivos documentales convencionales y fuentes secundarias como los materiales de libros, revistas, periódicos, etcétera.

2. Realizar un análisis del material proveniente de las entrevistas, pero sin dejar que el material testimonial hable por sí mismo, como si fuera un verdadera transcripción de lo real acontecido/ vivido. Esta parte del análisis debe responder a nuestras preguntas y problemas que se generaron desde un principio de la investigación. En este análisis deberemos tratar de comprender los silencios, lo implícito de la conversación, los acuerdos no verbales de la comunicación, el contexto de 1a narración.

3. Por último, el texto completo deberá confrontarse y triangularse con las demás fuentes orales y documentales a nuestra disposición. Esta lectura, posibilita una comprensión múltiple y relacional, no sólo en cuanto a la construcción del texto en sí mismo, sino también respecto a su posición y particularidad frente a los otros textos orales recopilados.

\section{(๑) $\Theta \odot$}

La Revista Estudios es editada por la Universidad de Costa Rica y se distribuye bajo una Licencia Creative Commons Atribución-NoComercial-CompartirIgual 3.0 Costa Rica. Para más información envíe un mensaje a 
Si bien no es recomendable privilegiar un modo de análisis en abstracto y generalizable, ya que cada proyecto, tiene sus particularidades, sus contextos, sus perfiles problemáticos, lo que si es conveniente es investigar sobre otras experiencias que se parezcan a nuestros proyecto y preguntas de investigación, para mejorar nuestros esfuerzos al intentar utilizar la evidencia oral.

\section{Utilización y difusión del archivo oral.}

Todo el material del proyecto será utilizado para generar artículos científicos que respondan a nuestro objetivo inicial, es decir, a explicar los cambios en el paisaje de los Centros Históricos del Turismo, sus repercusiones sobre los pobladores de estas zonas y a proporcionar información para la toma de decisiones, en materia de políticas públicas orientadas al desarrollo de un turismo sustentable, y que responda a las necesidades de las sociedades en que se inserta, tomando en cuenta el sentir de los grupos subalternos y propiciando verdaderas condiciones para el desarrollo económico, social y ambiental.

La recuperación del recuerdo y su transmisión, contribuye a forjar la memoria colectiva, que está en una permanente construcción y es a partir de un proyecto como este que, por medio de la entrevista de historia oral apelamos al recuerdo del entrevistado y en esa construcción del recuerdo vamos encontrando la memoria colectiva, por medio de la cual un pueblo recuerda un pasado que fue activamente transmitido a las generaciones contemporáneas que lo asumen como propio y pasan a formar parte de su autenticidad. Como señalan Barela, Miguez y García (2004)

"un pueblo olvida cuando la generación poseedora del pasado no lo transmite a la siguiente o ésta rechaza lo que recibió o cesa de transmitirlo, diferente de lo que ocurre en el plano de la psicología individual donde un individuo sólo puede recordar $u$ olvidar lo acaecido durante su propia existencia. De acuerdo con esto, la "memoria colectiva" podría definirse como ese movimiento dual de recepción y transmisión. Este movimiento es lo que forja la MEMORIA (mnemne) del grupo, lo que establece el continuo de su

\section{(C) $(0 \bigcirc)$}

La Revista Estudios es editada por la Universidad de Costa Rica y se distribuye bajo una Licencia Creative Commons Atribución-NoComercial-CompartirIgual 3.0 Costa Rica. Para más información envíe un mensaje a 
memoria. Memoria que, definida de esta manera, no incluiría un cúmulo de acontecimientos, fechas, referencias, sino que estaría formada por las tradiciones, ritos, valores, modos de relación, símbolos, creencias, que dan a un pueblo o grupo el sentido de su identidad y de su destino. (Barela et al, 2004, p. 17.)

La evidencia recopilada por medio de las entrevistas, al ser propiamente recabada y sistematizada, tiene detrás de sí un amplio el trabajo de investigación y se constituye en una fuente de información que al ser contrastada y complementada con la utilización de archivos y fuentes de información socio histórica convencionales se convierten en los elementos que esperamos dar a conocer por medio de los trabajos con la comunicación yo difusión de nuestros resultados. Para ello se espera realizar publicaciones en medios nacionales e internacionales.

\section{A modo de conclusión}

La historia oral como recurso metodológico ha sido poco utilizada en nuestro país para el estudio de los fenómenos históricos y los pocos casos que conocemos no han abordado el tema propuesto en nuestro estudio.

Cuando nos propusimos realizar estos apuntes sobre la metodología y el uso de la historia oral, partimos de la validez de la historia oral no sólo como una construcción de la fuente histórica, sino también como la posibilidad de recuperar a partir de la memoria individual o grupal un entramado de lazos sociales que reconoce en el anonimato cotidiano una actitud histórica.

La guía metodológica propuesta en este documento no pretende ser exhaustiva, más bien lo que busca es que el lector conozca el proyecto de investigación y la metodología que se seguirá para su desarrollo, basado en la oralidad, recogiendo la visión de pobladores de Puntarenas y Limón que han vivido y participado en la transformación de ese paisaje y que se han sido afectados y han afectado este proceso.

Nuestra intención es tratar de comprender, desde la visión de esos ciudadanos, hombres y mujeres, adultos y jóvenes, cómo interpretan su interacción con el

La Revista Estudios es editada por la Universidad de Costa Rica y se distribuye bajo una Licencia Creative Commons Atribución-NoComercial-CompartirIgual 3.0 Costa Rica. Para más información envíe un mensaje a 
medio y cuáles han sido las repercusiones en el ámbito económico, social, ambiental y cultural.

La información que sea recopilada con el proyecto, basados en la memoria, necesariamente tiene que considerar el papel que desempeña la transmisión oral, especialmente en los sectores sociales populares, ya que la comunicación al interior de estos grupos se hace utilizando este recurso y además los testimonios nos permitirán conocer no sólo los hechos que acontecieron, sino que nos aportan maneras de ver y pensar las cosas, valores, inquietudes, anhelos; en fin, una gama de creencias y pensamientos que acompañaron las experiencias de los entrevistados.

La información generada por la investigación servirá para construir la memoria histórica de estos pueblos, y a la vez proporcionará elementos para la toma de decisiones que permitan formular políticas públicas que permitan una mejor gestión del paisaje, visto como recurso que afecta la forma de vida de los pobladores y como recurso económico, en este caso para el turismo, capaz de ser utilizado para la mejora del bienestar de las comunidades.

Por ser un primer intento en este sentido, somos conscientes de que en su desarrollo habrá que realizar ajustes en la metodología y en la programación establecida, que será un proceso de aprendizaje en la práctica, pero estamos seguros de que los resultados serán valiosos, tanto para las comunidades, como para nosotros como investigadores.

En este sentido, el aporte de esta propuesta y las sugerencias metodológicas que aquí se han presentado tienen esa limitante ser formulada en teoría, pero a la vez, al estar relacionada con un proyectos y problemas concretos, nos permitirá ver si se adapta, se acopla y cumple con los objetivos del trabajo en desarrollo.

\section{Bibliografía consultada}

Aceves J. (2006). Historia Oral: ensayos y aportes de investigación. México. CIESAS.

\section{(c) (i) (2)}

La Revista Estudios es editada por la Universidad de Costa Rica y se distribuye bajo una Licencia Creative Commons Atribución-NoComercial-CompartirIgual 3.0 Costa Rica. Para más información envíe un mensaje a 
Aceves, Jorge. (1988). La historia oral y de vida: del recurso técnico a la experiencia de investigación. Técnicas de investigación en sociedad, cultura y comunicación. Madrid: Pearson Educación.

Bacazar, P. et al. (2005). Investigación cualitativa. México, UNAM.

Barela, L., Miguez, M., y García, L. (2004). Algunos apuntes sobre historia oral. Buenos Aires: Instituto Histórico de la Ciudad de Buenos Aires.

Cordero-Ulate, A. (2014). Paisajes y relatos de vida. Apuntes para la interpretación de los paisajes socioculturales con mención a Puntarenas y Limón (centro) en Costa Rica. México: Universidad Quintana Roo, Teoría y Praxis, Vol. 16, diciembre, 2014, pp. 9-31

Deslauriers, J. (1991). Recherche qualitative. Montreal. McGraw-Hill.

Fraser, R. (1993). "La Historia Oral como historia desde abajo" Revista AYER, 12, 79-92.

García, C., Vázquez, M., y Vilanova, M. (1988). Historia, fuente y archivo oral. En Actas del Seminario: Diseño de proyectos de historia oral. Madrid. Ministerio de Cultura.

Ginzburg, C. (2004). Memoria y globalización., Historia, Antropología y Fuentes Orales, 32, 29-40.

Gwyn, P. (1996). Historial oral. En Burke, P (Ed), Formas de hacer historia. Madrid: Alianza Editorial.

Joutard, P. (2007). Memoria e historia: ¿cómo superar el conflicto?, Revista Historia, Antropología y Fuentes Orales, 38, 115-122.

Meyer, E., y Olivera, A. (1971). La historia oral, origen, metodologías y perspectivas. Revista Colegio de México, 69, 372-387.

Nora, P. (1989). Between Memory and History: les Lieux de Memoire. Representations, 26, 7-24.

Parentini, L., y Contreras, H. Memorias vividas, memorias reconstruidas. Una propuesta metodológica para la utilización de las fuentes orales en la historia de la frontera mapuche. Revista Historia y Geografía. 22, 247-264.

\section{(c) (i) (2)}

La Revista Estudios es editada por la Universidad de Costa Rica y se distribuye bajo una Licencia Creative Commons Atribución-NoComercial-CompartirIgual 3.0 Costa Rica. Para más información envíe un mensaje a 
Revista Estudios, (39), 2019.

Diciembre 2019-Mayo 2020

ISSN 1659-3316

Bartels Villanueva Jorge

Portelli, A, (1991).The Death of Luigi Trastulli: Memory and the Event, idem, The Death of Luigi Trastulli and Other Stories: Form and Meaning in Oral History. New York: New York State University.

Portelli, A. (2004). What makes oral history different? The Oral History Reader. London: Routledge R. Perks and A. Thomson.

Argueta B., Arrieta C., Bartels J., Carballo P., Chen S., Cordero A., Guardia D. y Lepe I.,(2012). Proyecto de investigación: "Centros Históricos del Turismo: Puntarenas y Limón" inscrito en la Vicerrectoría de Investigación de la Universidad de Costa Rica.

Thompson, P. (1978). Voice of the Past. Oxford: University Press.

Thompson, P. (1988). La voz del pasado. Historia Oral, Valencia, Edicions Alfons El Magnimim, 1-338.

Unesco (2002). Paisajes culturales en Mesoamérica: Memoria. Reunión de Expertos sobre los Paisajes Culturales en Mesoamérica. San José de Costa Rica: Oficina de la Unesco para América Central.

La Revista Estudios es editada por la Universidad de Costa Rica y se distribuye bajo una Licencia Creative Commons Atribución-NoComercial-CompartirIgual 3.0 Costa Rica. Para más información envíe un mensaje a 